\title{
Application of Ginkgo Biloba L. Extract at Early Stage of Tumor Development Helps Cyclophosphamide Inhibit the Growth of Tumor Cells
}

\section{Dawei Chen}

Jilin University School of Public Health

Jinghui Yang

China-Japan Union Hospital of Jilin University

\section{Fanzhu Meng}

Jilin University School of Public Health

\section{Cheryl L. Beseler}

Department of Psychology Coloradao State University

Hao Li

Jilin University School of Public Health

Xiaomei Liu

Jilin University School of Public Health

Yinpei Guo

Jilin University School of Public Health

\section{Lili Qin}

Jilin University School of Public Health

\section{Ruyue Zuo}

Jilin University School of Public Health

Shuping Ren ( $\nabla$ rensp@jlu.edu.cn )

Jilin University School of Public Health https://orcid.org/0000-0002-1212-9528

\section{Research article}

Keywords: antioxidant, tumor inhibition, survival analysis, Ginkgo biloba L. extract, cyclophosphamide, combination

Posted Date: August 26th, 2020

DOI: https://doi.org/10.21203/rs.3.rs-50132/v1 
License: (c) (i) This work is licensed under a Creative Commons Attribution 4.0 International License. Read Full License 


\section{Abstract}

Background There is inconsistency in the application of antioxidants in tumor treament, the study is to explore whether the application of an antioxidant at the early stage of tumor transplantation enhances efficacy of a chemotherapeutic agent in inhibiting tumor cell growth.

Methods EMT- 6 cells were injected into the 48 ICR mice. 24h later, cyclophosphamide (CTX) and Ginkgo biloba L. extract (GBE) were administered to mice separately and in combination. Apoptotic markers and related signaling pathways were measured. Tumor weights were compared and survival analysis was used to investigate latency periods in the three treatment groups compared to a PBS control group.

Results The mice administered GBE and CTX had significantly lower tumor weights compared with those administered PBS ( $p=0.01)$, however, the mice administered CTX did not have significantly lower tumor

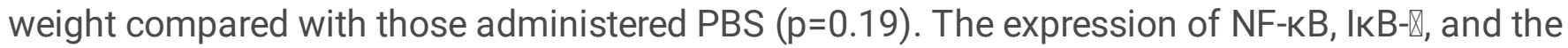

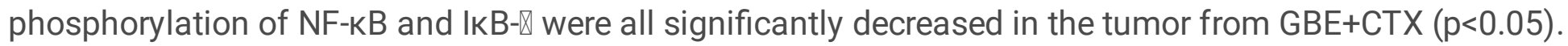
Moreover, the ratio of $\mathrm{Bax} / \mathrm{Bcl}-2$ was significantly higher for the tumor of mice administered $\mathrm{GBE}+\mathrm{CTX}$ than that of mice administered GBE, CTX, or PBS $(p<0.05)$, the expression of FADD, Caspase-8, and Cyto-C was significantly increased in the tumors of mice administered GBE+CTX than those administered PBS or GBE $(p<0.05)$.

Conclusions Application of an antioxidant at an early stage of tumor transplantation could help chemotherapeutic agent inhibit the growth of tumor cells. GBE+CTX treated mice showed a longer latency period to tumor development and markers that consistently indicated greater apoptotic activity and reduced tumor promotion.

\section{Background}

Globally, breast cancer is the second major cause of cancer death among women [1]. The currently available treatment strategies are removal of the tumor followed by chemotherapy, radiotherapy, or other affiliated therapy based on the clinical characteristics of the patient [2]. Cyclophosphamide (CTX) is a common chemotherapeutic agent for breast cancer patients who have undergone surgery. CTX metabolites act by disrupting the DNA and arrests the cell cycle of tumor cells, which results in their apoptosis or growth inhibition [3]. However, CTX triggers the production of excessive amounts of reactive oxygen species (ROS), which will produce a variety of side effects in different organs or systems $[4,5]$. Moreover, ROS is involved in all stages of cancer development with modest levels of ROS promoting tumors and higher levels suppressing tumors, all in stages that vary tremendously [6]. In the initiation stage of cancer, ROS may promote the expansion of cancerous cells by modifying the genes related to apoptosis [7], upregulating antiapoptotic genes and downregulating proapoptotic proteins via PI3K/AKT and ERK/MEK pathways [8]. Furthermore, ROS will activate the NF-KB pathway and NF-KB-dependent genes that are involved in apoptosis and cell adhesion, implicated in multiple aspects of cancer 
development [9]. ROS, as a survival or apoptotic signal to cancer cells, is determined by the dosage, duration, type, and site of ROS production [10].

Previous studies suggest that antioxidants are able to produce protective effects against several types of cancers including breast cancer [11] and are also effective in reducing breast cancer recurrence for patients on tamoxifen [12]. Thus, we hypothesized that antioxidation might produce inhibitory effects on the growth of EMT-6 cells, a murine mammary carcinoma cell line, at the early stage and the combination of Ginkgo biloba L. extract (GBE) and CTX would have better inhibiting effects on the growth of EMT-6 cells when compared to EMT-6 cells treated with CTX alone.

\section{Methods}

\subsection{Cells and reagents}

EMT-6 cells were a gift from the Department of Molecular Biology, College of Basic Medicine, Jilin University. ICR female mice were purchased from the Liaoning Changsheng Biotechnology, Co. Limited, China. GBE was produced by Chi Sheng Chemical Corporation, Taiwan.

\subsection{EMT-6 Cell culture}

EMT-6 cells were incubated in RPMI 1640 medium supplemented with 10\% fetal bovine serum (FBS, Hyclone USA), penicillin $100 \mathrm{IU} / \mathrm{mL}$, streptomycin $100 \mathrm{ug} / \mathrm{mL}$ at $37^{\circ} \mathrm{C}$ with $5 \% \mathrm{CO}_{2}$.

\subsection{Animal experiment}

To investigate whether the application of GBE at early state of tumor development could help CTX inhibit the growth of EMT-6 cells, we administered GBE to the mice after EMT-6 cells were injected to the mice for 24 hours. All procedures used in handling animals were approved by the Institutional Review Board of the School of Public Health, Jilin University. ICR mice were housed in the animal center for ten days to adapt to the environment. After adaptation, the mice were injected with EMT- 6 cells at the concentration of $1 \times$ $10^{6} \mathrm{cell} / \mathrm{s} /$ mouse in the right thigh followed by random distribution of these mice into 4 treatment groups of 12 mice per group. The groups included PBS, GBE, CTX, and GBE + CTX. After $24 \mathrm{~h}$, the mice in the PBS group were injected with PBS intraperitoneally at a volume of $0.2 \mathrm{ml}$; the mice in the GBE group were injected with GBE intraperitoneally at the concentration of $3 \mathrm{mg} / \mathrm{kg}$ body weight; the CTX mice were injected intraperitoneally with CTX at the dose of $2 \mathrm{mg} / \mathrm{kg}$ body weight; the mice in the GBE + CTX group were injected intraperitoneally with both CTX at the concentration of $2 \mathrm{mg} / \mathrm{kg}$ body weight and GBE at the concentration of $3 \mathrm{mg} / \mathrm{kg}$ body weight. The injections were conducted consecutively for 10 days when all the mice in the PBS group developed a palpated tumor, except for the CTX group which was injected every other day. A fifth group of 12 mice (negative controls) were not injected with EMT-6 cells but were included to compare body weight gain and serum malondialdehyde (MDA) and superoxide dismutase (SOD) levels in cancer-free mice. Euthanasia: All mice were anesthetized with $10 \%$ chloral hydrate before the mice were sacrificed. The changes of body weight, the latency of tumor onset, the growth of tumor, 
tumor weight, survival analysis, serum MDA levels and SOD activities, and the key elements in the NF-KB signaling pathway, $\mathrm{PI} 3 \mathrm{~K} / \mathrm{AKT}$ pathway and apoptosis were measured and compared among different groups.

\subsection{Body weight measurement}

Body weight is a basic parameter of body status. The measurement of body weight would help learn how the growth of tumor affect the mice. The body weight of the mice was measured every day until the mice were sacrificed. The growth trends of body weight were compared among treatment groups and the control group.

\subsection{Tumor onset and growth}

To explore whether GBE and CTX would prolong the onset of tumor and inhibit the growth of EMT-6 cells, we determined the tumor onset and tumor growth. Tumor onset was determined by palpation. The site of tumor injection was observed and palpated each day. The day of tumor onset was recorded for each and every mouse to compare the tumor latency among the different groups. The length and width of the tumor was measured. The tumor volume was calculated based on the formula $V=\left(a^{\star} b^{2}\right) / 2$, where $a$ is the length of the tumor and $b$ is the width of the tumor. When each and every mouse in the PBS group developed a tumor, all the mice from different groups were sacrificed and the tumor from each mouse was removed and weighed. Tumor weight was compared among the four groups.

\subsection{Determination of malondialdehyde (MDA) and superoxide dismutase (SOD) in serum}

Tumor growth is closely associated with ROS production which is also a underlying mechanism of tumor growth, we measured the level of MDA and activities of SOD in the serum. The measurement of serum MDA and SOD was described in detail in Ren et al [13] in each mouse in the four treatment groups and in the untreated control mice.

\subsection{Western blot}

NF-KB signaling pathway, PI3K/AKT pathway and apoptosis are implicated in the multiple aspects of tumor development. To reveal the mechanisms underlying GBE and CTX inhibiting the growth of EMT-6 cells, we determined the expression of key elements in the NF-KB signaling pathway, PI3K/AKT pathway and apoptosis. Western blot analysis was conducted as in the protocols described in Ren et al [13]. Briefly, equal amounts of protein $(20 \mu \mathrm{g} /$ well $)$ were loaded and separated by $10 \%$ SDS-polyacrylamide gel electrophoresis and transferred to polyvinylidene difluoride (PVDF) membranes. The membrane was incubated with rabbit anti-NF-KB(1:1000, CST USA), anti-p-NF-KB (1:1000, CST USA), anti-PI3K (1:1000, CST USA), anti-Akt (1:1000, CST USA), anti-IKB (1:1000, CST USA), anti-caspase 8 (1:1000, CST USA), anti-CytC (1:1000, Santa Crue Biotechnology, USA), anti-FADD (1:1000, Santa Cruz Biotechnology, USA), anti-p53 (1:1000, Santa Cruz Biotechnology, USA), anti-Bax (1: 500, Bioss China), anti-Bcl-2 (1:500, Bioss China), anti- $\beta$-actin (1:1000, CST, USA), and anti-rabbit IgG conjugated with HRP (1: 6600, Jackson IR, USA). Detection was done by measuring the chemiluminescence of ECL reagent (PIERCE, USA). The 
photographs generated were quantitatively analyzed with a Quantity One image densitometer. Protein levels were standardized by comparison with anti- $\beta$-actin antibody.

\subsection{Statistical analysis \\ 2.8.1 ROS biochemical analyses}

Results are presented as mean \pm SD. SPSS 21.0 software was used to do the statistical analysis by ANOVA and Duncan multiple comparisons were carried out for comparison between groups. A $p<0.05$ was considered significant.

\subsubsection{Differences in median tumor weights and latency times between treatment groups}

Normality was tested using quantile plots. We used a Kruskal-Wallis statistic to generate a 100,000 bootstrapped samples from which to estimate the $p$-value for testing for overall differences among groups. Pairwise comparisons of medians were conducted with an exact Wilcoxon statistic and a Dunn's test was used to adjust for multiple comparisons.

\subsubsection{Survival analysis}

We tested whether the data met the proportional hazards assumption and used the Cox proportional hazards model to assess differences in time-to-event analysis with uncensored data because all outcomes were observed at 10 days. The four treatments groups were coded as $1=P B S, 2=C T X, 3=G B E$ and $4=\mathrm{GBE}+\mathrm{CTX}$. Exponentiated hazard ratios (HR) with 95\% confidence intervals (Cl) were calculated. A HR less than 1.0 indicates that the lower coded value demonstrated a shorter latency period. We used the method of Peto and Peto (1972), a modified log rank test, to generate the $p$-value because it works well in small samples and is better at detecting early differences in survival. We plotted hazard curves for the treatment groups [14].

\section{Results}

\subsection{Body weight comparison in mice of different groups}

The body weight of mice from different groups tended to grow with time. When the mice were sacrificed, there was no significant difference in the body weight of mice among different groups (Fig. 1), which indicates EMT-6 cell growth did not affect the growth of the mice.

\subsection{Comparison of tumor onset}

No significant differences were identified in the overall median latency times $(p=0.20)$. Similarly, no differences were observed for CTX compared to PBS $(p=0.32)$, GBE compared to PBS $(p=0.40)$, or CTX + GBE compared to PBS $(p=0.06)$. 


\subsection{Tumor weight comparison of mice from different groups}

The tumor weights in the PBS control group were normally-distributed; the other groups demonstrated non-normality. Overall, tumor weights did not differ by treatment group $(p=0.20)$. Despite the lack of significance in the overall group effects, we did a pairwise comparison to test whether the CTX $+\mathrm{GBE}$ group differed from the PBS group. Comparing each treatment group to the PBS group showed that the CTX-treated group $(p=0.19)$ and the GBE-treated group $(p=0.15)$ did not differ from the PBS control, however, the CTX + GBE group differed significantly from the PBS control group $(p=0.01)$. Adjusted for multiple testing produced $p$-values of $0.30,0.18$, and 0.07 , respectively. Tumor volume and tumor picture were shown in Fig. 2.

\subsection{Survival analysis}

The data met the proportional hazards assumption. The effect size for the treatment effect on the latency period was 0.84 (Cl: 0.65, 1.09, standard error $(\mathrm{SE})=0.13, p=0.32)$. See (Fig. 3 ).

Comparing the GBE + CTX group to the PBS group showed a treatment effect of $0.81(\mathrm{Cl} 0.62,1.06, \mathrm{SE}=$ $0.14, p=0.067)$. Figure 4 shows a rightward shift in the GBE + CTX group, indicating that co-treatment with $\mathrm{GBE}+\mathrm{CTX}$ resulted in greater time to tumor detection.

\subsection{Comparison of MDA and SOD levels in serum}

All mice from the GBE, CTX and GBE + CTX groups showed lower levels of MDA and higher activities of SOD than those from the PBS group. The mice from the GBE + CTX group and the GBE group had lower levels of MDA and higher SOD activities than those from PBS or CTX group (Fig. 5A, B) $(p<0.05)$.

\subsection{Expression of NF-kB, IkB, Bax, Bcl-2, FADD, Caspase-8, PI3K, Akt, and P 53}

The expression of NF-KB and IKB- $₫$ was significantly decreased in the tumor of mice treated with GBE + CTX compared with mice treated with GBE, CTX, or PBS $(p<0.05)$ ( Fig. 6A), and the phosphorylation of NF-кB and ІкB- $\$ was also significantly decreased (Fig. 6A, B). Similarly, the expression of p53 was significantly increased in the tumor of mice treated with GBE + CTX compared with mice treated with GBE or PBS $(p<0.05)$ (Fig. 6F).

The expression of Bax was significantly increased in the tumor of mice treated with GBE + CTX compared with those treated with GBE, CTX, or PBS (Fig. 6C). In addition, the Bcl-2 expression was significantly decreased in the tumor of mice treated by GBE + CTX compared with mice treated with GBE, CTX, or PBS $(p<0.05)$ (Fig. 6C). The ratio of Bax/Bcl-2 was significantly higher for the tumor of mice treated by GBE + CTX than that of mice treated with GBE, CTX, or PBS ( $p<0.05)$. (Fig. 6D) 
The expression of FADD, Caspase-8, and Cyto-C was significantly increased in the tumors of mice treated with GBE + CTX than those treated with PBS or GBE $(p<0.05)($ Fig. $6 \mathrm{C})$.

The expression of PI3K and Akt in the tumors of mice treated with GBE + CTX was only significantly decreased when compared to those treated with PBS $(p<0.05)$ (Fig. 6E).

\section{Discussion}

The main finding of our study is that in mice injected with EMT-6 cells, treatment with a combination of GBE and CTX produced better growth inhibition of tumors compared to PBS-treated mice. GBE or CTX alone did not produce a better inhibition on the growth of EMT-6 cells than PBS. Although the results of the survival analysis did not reach statistical significance, a clear upward shift was seen in the survival curves in the GBE + CTX group compared to the PBS controls. Furthermore, the median latency time comparing the GBE + CTX group to the PBS control group with $p=0.06$ suggests that statistical significance likely would have been achieved with a larger sample size. Additional assessment of indicators of ROS and apoptotic pathways supported these results. This finding is important because it might offer a treatment regimen for prevention of tumor recurrence.

As expected, MDA levels were higher and SOD activities lower in PBS-treated mice compared to untreated, control mice because cancers cells produce higher levels of ROS $[15,16]$. MDA was lower and SOD activity higher in the GBE group compared to the PBS group, demonstrating the antioxidant effects of GBE. CTX showed opposite effects of GBE on MDA and SOD but adding GBE to CTX caused the mice in the combination group to look similar to the GBE group. These comparisons support previous observations that cancer cells and CTX both increase ROS and provides evidence that GBE can reduce ROS with and without CTX present.

Although there is a balance between the benefits and disadvantages of adjuvant antioxidants in chemotherapy, early co-administration of antioxidants with chemotherapeutic agents might be useful in scavenging ROS. The resulting reduction in ROS signaling pathways may, in early stages, suppress tumor growth. Our results showed that GBE + CTX reduced IKB expression and its phosphorylation above that of CTX alone, resulting in decreased activation of the NF-KB signaling pathway and promoting apoptosis. The pro-death response to GBE + CTX was consistent in the increased expression of FADD, Caspase-8, Cyto-C, Bax and Bax/Bcl-2 ratio showing the highest levels in the PBS group, followed by the GBE, CTX and the GBE + CTX groups. A reverse pattern was observed for expression of Bcl-2. These patterns of expression support the hypothesis that GBE acts to promote apoptosis in the early stage of tumor initiation and enhance the effects of CTX.

Downstream of the PI3-K/Akt pathway is p65 where phosphorylation in the IKK-dependent pathway leads to activation of NF-KB, resulting in the promotion of carcinogenesis $[8,17]$. Decreased PI3-K/Akt pathway activity promotes autophagy-mediated cell death $[18,19]$. Autophagy is initiated in part by genotoxic stress with an increased expression of p53 [20-22], and our results showed an increase in p53 in the GBE + CTX group compared to the PBS group. 
In summary, in our study CTX and GBE was administered to the mice after the tumor cells were transplanted into the mice after only 24 hours, which was the initial stage of tumor growth. There is inconsistency regarding whether antioxidation should be used in tumor treatment [10]. Tumor weight was significantly decreased by CTX + GBE. The mechanisms underlying this change are that GBE +CTX significantly inhibited the activation of NF-kB, upregulated the proapoptotic and P53 genes, and downregulated the PI3K/Akt pathway, which are implicated into the expansion of cancerous cells. It has been reported that ROS will promote the growth of tumor cells by triggering one of the cell growth signaling pathways, NF-kB. GBE is able to remove ROS which can activate NF-kB, as shown in our findings. The expression and phosphorylation of NF-kB were both significantly suppressed in the GBE + CTX group. Moreover, ROS is also able to promote the expansion of cancerous cells by modifying the genes related to apoptosis and to upregulate antiapoptotic genes and downregulate proapoptotic proteins via PI3K/AKT and ERK/MEK pathways [16]. GBE + CTX is also able to increase the expression of genes related to apoptosis Bax, FADD, Caspase-8, and Cyto-C. Furthermore, PI3K/AKT was significantly decreased by GBE + CTX.

The strength of the present study is that we conducted an experiment to investigate whether or not, when the tumor cells was already present in the body, at the early stage of tumor development, chemotherapy and antioxidation would produce a better inhibition on the growth of tumor cells. The findings of our study suggest that CTX alone would not inhibit the growth of EMT- 6 cells better than PBS, or GBE, indicating that when tumor cells were present, chemotherapy would not be potent enough to kill the tumor cells inside body. After surgery, antioxidation and chemotherapy might be helpful to fight the recurrence or slow the growth of tumor cells, our findings are in accordance with Thomson's et al' [12].

A weakness of the present study is that the tumor cells were injected into the mice; the procedure may not simulate the development of tumor onset in the real situation. All of the mice had developed palpated tumors on or before day 10 in our experiment. Had we continued the trial period, we might have observed a significant difference in tumor weights between CTX and GBE + CTX treated groups.

\section{Conclusion}

In this preliminary study of using GBE in combination with CTX, we showed several programmed cell death pathways were activated. Consistently, GBE+CTX treated mice showed a longer latency period to tumor development and markers that consistently indicated greater apoptotic activity and reduced tumor promotion. Although additional studies are needed, this study provides a potential avenue for preventing breast cancer recurrence in women being treated with cyclophosphamide after tumor removal surgery. Furthermore, it provides a direction for future research on the role of antioxidation in conjunction with chemotherapy.

\section{Abbreviations}

Ginkgo biloba L. extract, GBE 
Cyclophosphamide, CTX

Malondialdehyde, MDA

Superoxide dismutase, SOD

\section{Declarations}

\section{Ethics approval and consent to participate:}

All procedures used in handling animals were approved by the Institutional Review Board of the School of Public Health, Jilin University.

\section{Consent for publication:}

Not applicable

\section{Availability of data and materials:}

Data sharing is not applicable to this article as no datasets were generated or analysed during the current study.

\section{Competing interests:}

The authors declare that they have no competing interests.

\section{Funding:}

This study was funded by Leshiguang Measurement Technology Corporation Limited (Grant No.2018YX046). The funding body has on role in the design of the study and collection, analysis, and interpretation of data and in writing the manuscript.

\section{Authors' contributions:}

Conception and Design: DWC, JHY, SPR; Formal Analysis and Interpretation: CLB, SPR; Investigation: JHY, FZM, HL, YPG, LLQ, RYZ; Writing - Original Draft Preparation: DWC and SPR; Writing - Review \& Editing: CLB and SPR; Funding Acquisition: DWC, SPR. All authors approved the final manuscript.

\section{Acknowledgements:}


We would like to thank Professor Liying Wang at the Department of Molecular Biology, College of Basic Medicine, Jilin University, for her gift of EMT-6 cells.

\section{References}

1. Azim HA, Ibrahim AS. Breast cancer in Egypt, China and Chinese: statistics and beyond. J Thorac Dis. 2014;6(7):864-6. doi:10.3978/j.issn.2072-1439.2014.06.38.

2. $10.1016 /$ j.semcancer.2019.12.008

Tewari D, Patni P, Bishayee A, Sah AN, Bishayee A. Natural products targeting the PI3K-Akt-mTOR signaling pathway in cancer: A novel therapeutic strategy. Semin Cancer Biol. 2019; S1044-579 × (19)30405-5. doi:10.1016/j.semcancer.2019.12.008.

3. Emadi A, Jones RJ, Brodsky RA. Cyclophosphamide and cancer: golden anniversary. Nat Rev Clin Oncol. 2009;6(11):638-47. doi:10.1038/nrclinonc.2009.146.

4. Kurauchi K, Nishikawa T, Miyahara E, Okamoto Y, Kawano Y. Role of metabolites of cyclophosphamide in cardiotoxicity. BMC Res Notes. 2017;10(1):406. doi:10.1186/s13104-017-27262.

5. Bhattacharjee A, Basu A, Ghosh P, Biswas J, Bhattacharya S. Protective effect of Selenium nanoparticle against cyclophosphamide induced hepatotoxicity and genotoxicity in Swiss albino mice. J Biomater Appl. 2014;29(2):303-17. doi:10.1177/0885328214523323.

6. Gupta SC, Hevia D, Patchva S, Park B, Koh W, Aggarwal BB. Upsides and downsides of reactive oxygen species for cancer: the roles of reactive oxygen species in tumorigenesis, prevention, and therapy. Antioxid Redox Signal. 2012;16(11):1295-322. doi:10.1089/ars.2011.4414.

7. Trueba GP, Sánchez GM, Giuliani A. Oxygen free radical and antioxidant defense mechanism in cancer. Front Biosci. 2004;9:2029-44. doi:10.2741/1335. Published 2004 Sep 1.

8. McCubrey JA, Steelman LS, Chappell WH, Abrams SL, Wong EW, Chang F, Lehmann B, Terrian DM, Milella M, Tafuri A, Stivala F, Libra M, Basecke J, Evangelisti C, Martelli AM, Franklin RA. Roles of the Raf/MEK/ERK pathway in cell growth, malignant transformation and drug resistance. Biochim Biophys Acta. 2007;1773(8):1263-84. doi:10.1016/j.bbamcr.2006.10.001.

9. Liu Z, Ren Z, Zhang J, Chuang C-C, Kandaswamy E, Zhou T, Zuo L. Role of ROS and Nutritional Antioxidants in Human Diseases. Front Physiol. 2018;9:477. doi:10.3389/fphys.2018.00477.

10. Yang $H$, Villani RM, Wang $H$, Simpson MJ, Roberts MS, Tang M, Liang X. The role of cellular reactive oxygen species in cancer chemotherapy. J Exp Clin Cancer Res. 2018;37(1):266.

doi:10.1186/s13046-018-0909-x.

11. Turati F, Rossi M, Pelucchi C, Levi F, La Vecchia C. Fruit and vegetables and cancer risk: a review of southern European studies. Br J Nutr. 2015;113(Suppl 2):102-10.

doi:10.1017/S0007114515000148.

12. Thomson CA, Rock CL, Thompson PA, Caan BJ, Cussler E, Flatt SW, Pierce JP. Vegetable intake is associated with reduced breast cancer recurrence in tamoxifen users: a secondary analysis from the 
Women's Healthy Eating and Living Study. Breast Cancer Res Treat. 2011;125(2):519-27. doi:10.1007/s10549-010-1014-9.

13. Ren M, Yang S, Li J, Hu Y, Ren Z, Ren S. Ginkgo biloba L. extract enhances the effectiveness of syngeneic bone marrow mesenchymal stem cells in lowering blood glucose levels and reversing oxidative stress. Endocrine. 2013;43(2):360-9. doi:10.1007/s12020-012-9745-5. Epub 2012 Jul 20.

14. Peto R, Peto J. Asymptotically efficient rank invariant test procedures. J Royal Stat Soc. 1972;135:186-207.

15. Behrend L, Henderson G, Zwacka RM. Reactive oxygen species in oncogenic transformation. Biochem Soc Trans. 2003;31:1441-4. doi:10.1042/bst0311441.

16. Toyokuni S, Okamoto K, Yodoi J, Hiai H. Persistent oxidative stress in cancer. FEBS Lett. 1995;358:13. doi:10.1016/0014-5793(94)01368-b.

17. Madrid LV, Mayo MW, Reuther JY, Baldwin AS Jr. Akt stimulates the transactivation potential of the RelA/p65 Subunit of NF-kappa B through utilization of the lkappa B kinase and activation of the mitogen-activated protein kinase p38. J Biol Chem. 2001;276(22):18934-40. doi:10.1074/jbc.M101103200. Epub 2001 Mar 20.

18. $10.1074 /$ jbc.M110572200

Sizemore N, Lerner N, Dombrowski N, Sakurai H, Stark GR. Distinct roles of the Ikappa B kinase alpha and beta subunits in liberating nuclear factor kappa B (NF-kappa B) from Ikappa B and in phosphorylating the p65 subunit of NF-kappa B. J Biol Chem. 2002; 8;277(6):3863-9. doi: 10.1074/jbc.M110572200. Epub 2001 Dec 3.

19. Yu X, Long YC, Shen HM. Differential regulatory functions of three classes of phosphatidylinositol and phosphoinositide 3-kinases in autophagy. Autophagy. 2015;11(10):1711-28. doi:10.1080/15548627.2015.1043076.

20. Degtyarev M, De Mazière A, Orr C, Lin J, Lee BB, Tien JY, Prior WW, van Dijk S, Wu H, Gray DC, Davis DP, Stern HM, Murray LJ, Hoeflich KP, Klumperman J, Friedman LS, Lin K. Akt inhibition promotes autophagy and sensitizes PTEN-null tumors to lysosomotropic agents. J Cell Biol. 2008;183(1):10116. doi:10.1083/jcb.200801099.

21. Budanov AV, Karin M. p53 target genes sestrin1 and sestrin2 connect genotoxic stress and mTOR signaling. Cell. 2008;134:451-60. doi:10.1016/j.cell.2008.06.028.

22. Maiuri MC, Malik SA, Morselli E, Kepp O, Criollo A, Mouchel PL, Carnuccio R, Kroemer G. Stimulation of autophagy by the p53 target geneSestrin2. Cell Cycle. 2009;8:1571-6. doi:10.4161/cc.8.10.8498. Epub 2009 May 20.

\section{Figures}




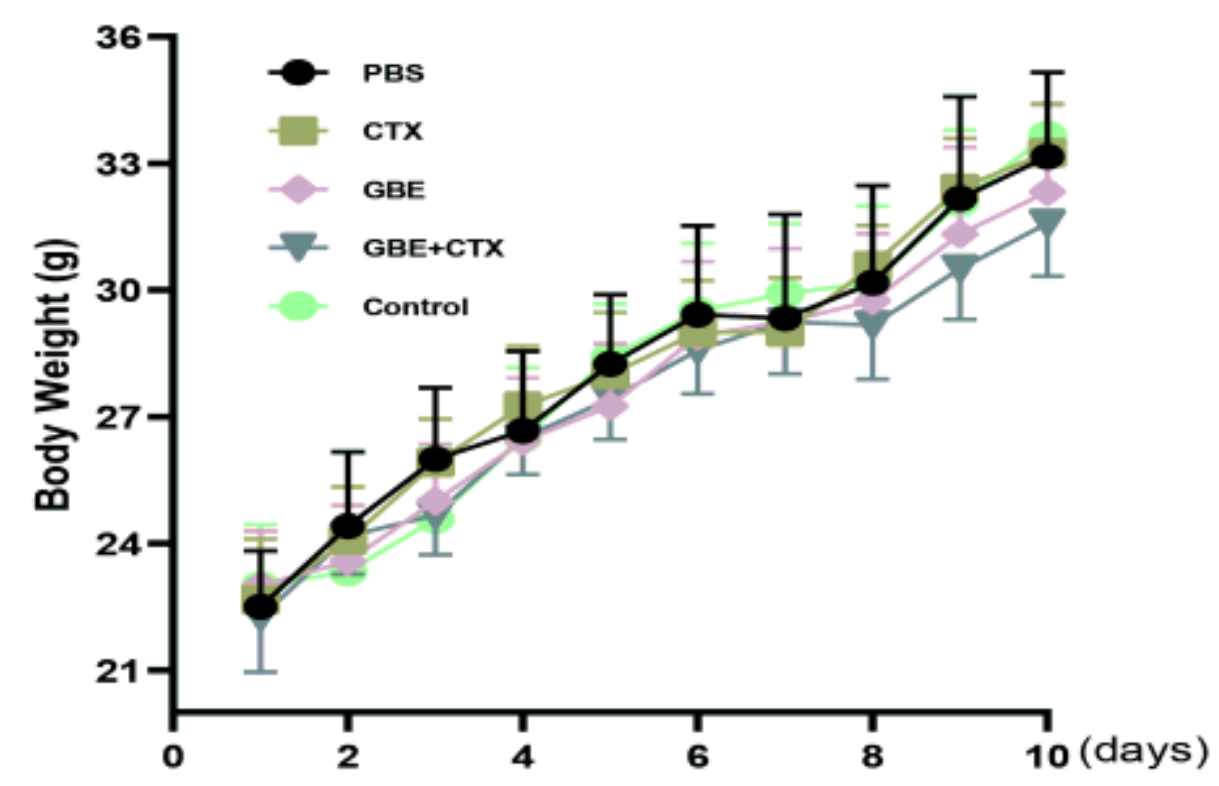

Figure 1

Body weight change of mice in different groups The body weights of the mice were measured every day until all mice in the PBS group grew palpated tumors and all mice were sacrificed.

A

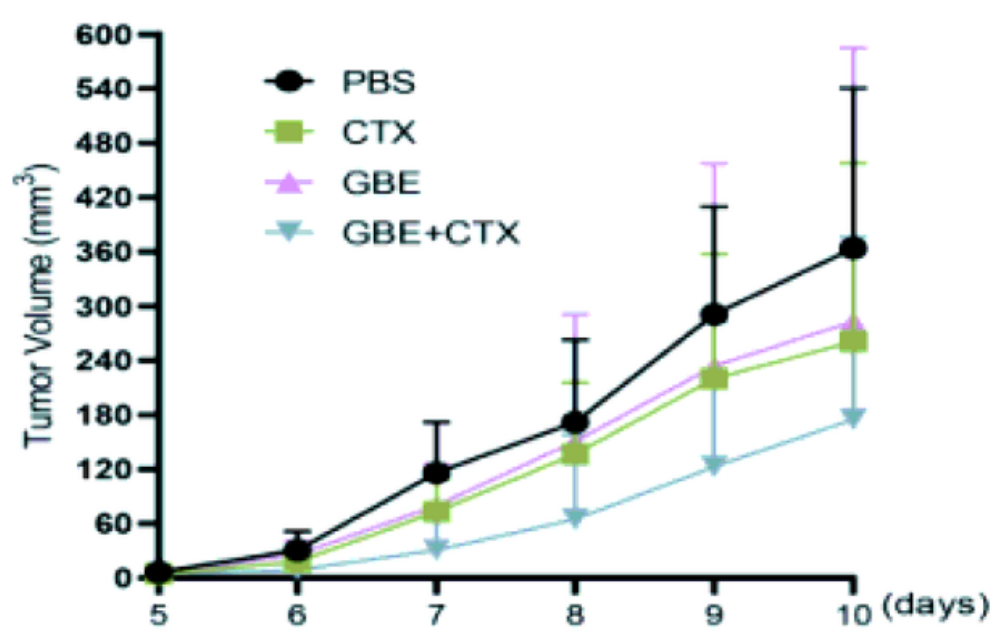

B

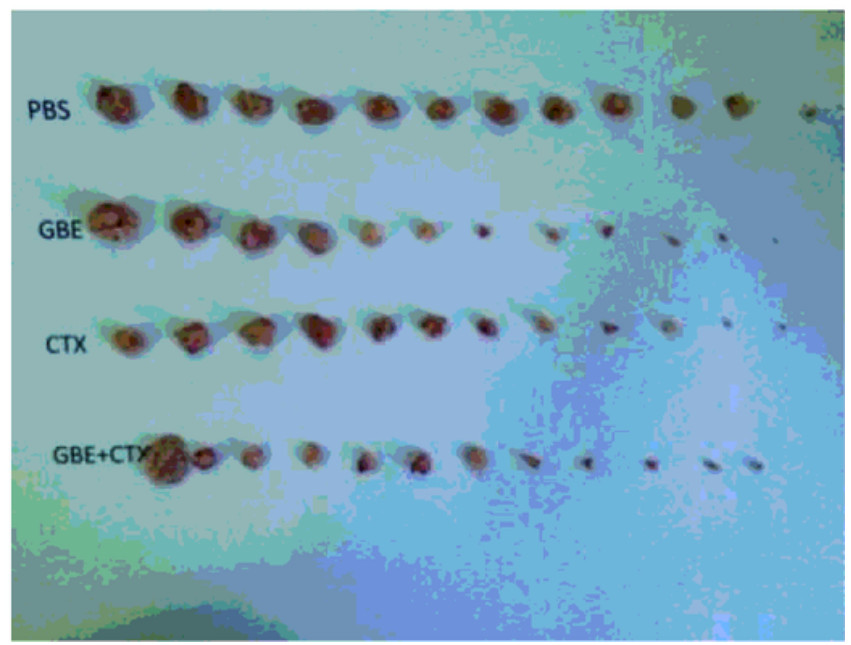

\section{Figure 2}

Comparison of tumor volume and weight in mice from different groups The length and width of the tumor was measured every day. On day 10, all the mice from the PBS developed a palpated tumor and all the mice from different groups were anesthetized and sacrificed, the tumor was removed from each and every mice $(n=12)$. A. Tumor growth represented by tumor volume, $n=12$. B. Tumor picture. 


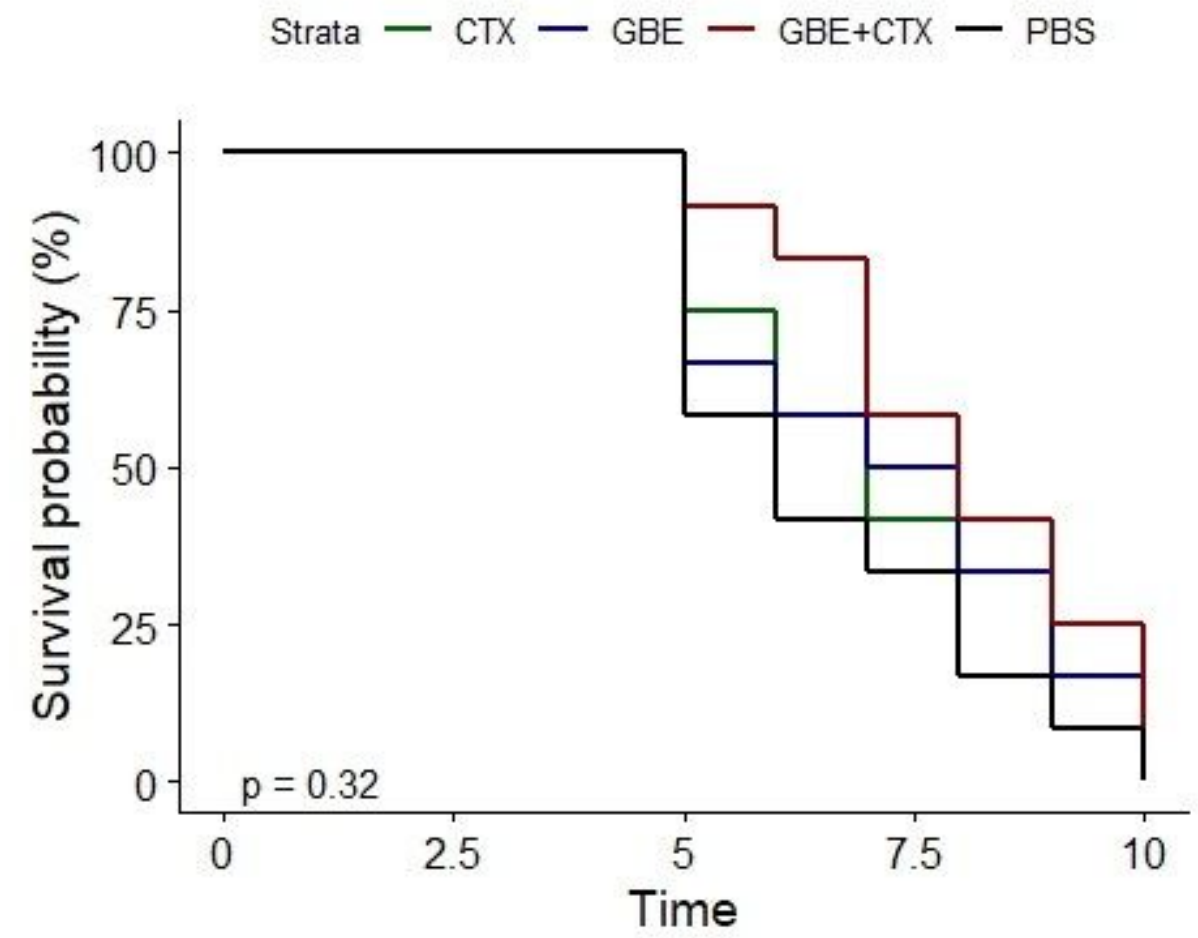

Figure 3

Survival curves for four treatment groups with 12 per group

$$
\text { Strata - GBE+CTX - PBS }
$$

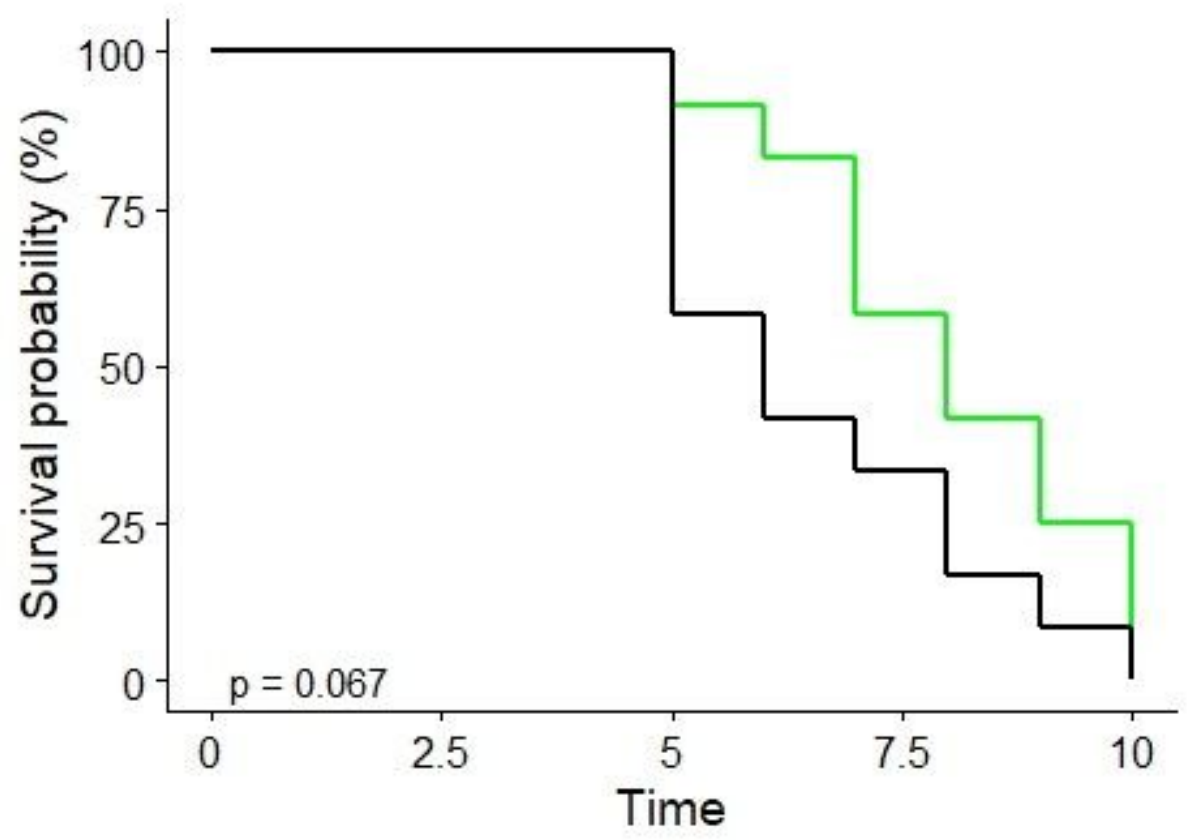

Figure 4

Survival curves comparing the combined $C T X+G B E$ group to the PBS control group 
A

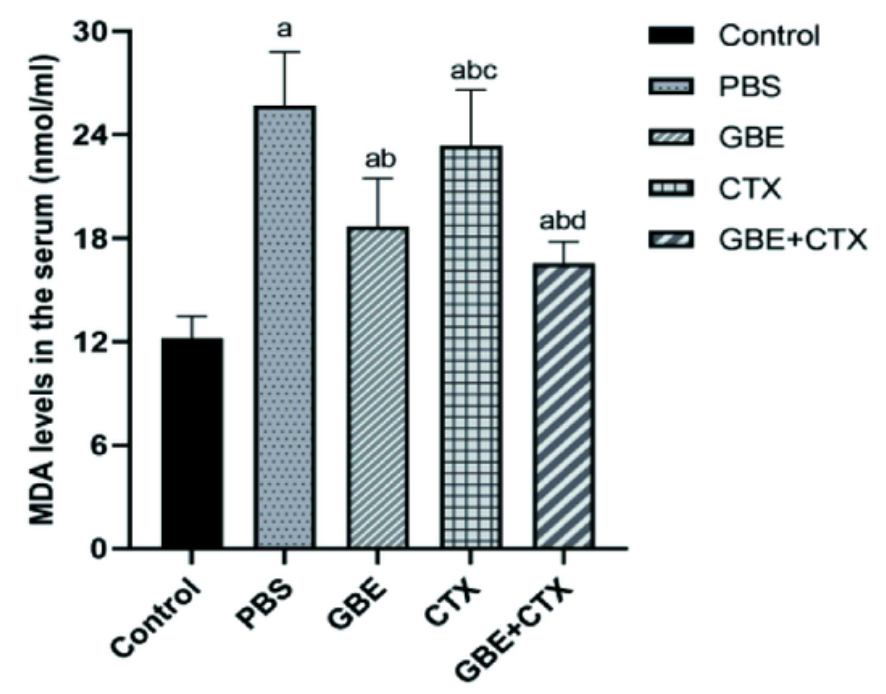

B

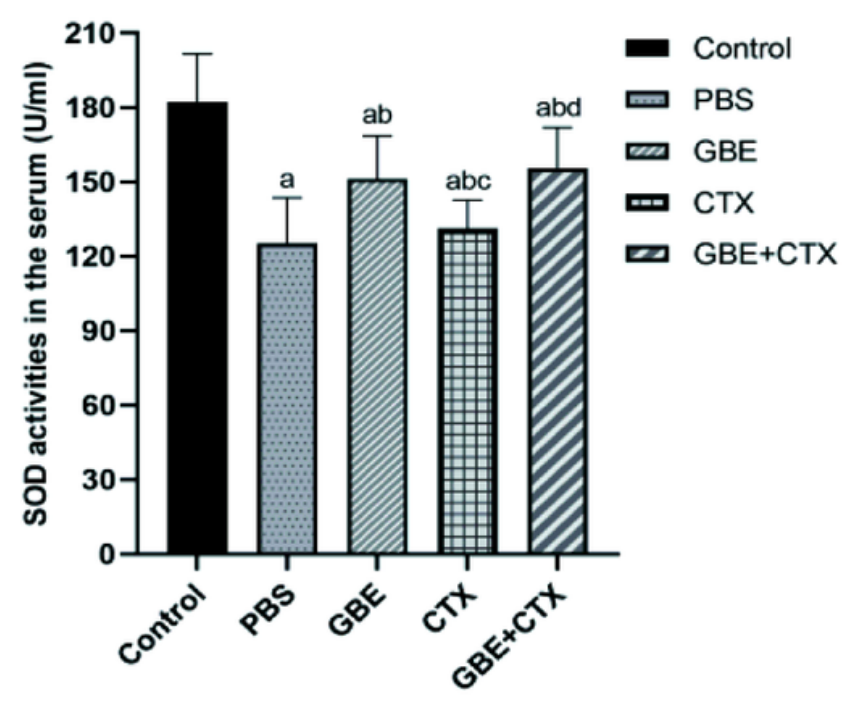

Figure 5

5 Comparison of MDA levels and SOD activities in the serum of mice A. Comparison of MDA levels in the serum of mice from different groups. B Comparison of SOD activities in the serum of mice from different groups. ap $<0.05$ vs control; $b p<0.05$ vs $P B S ; c p<0.05$ vs $\mathrm{GBE} ; \mathrm{dp}<0.05$ vs $\mathrm{CTX}$ 
A

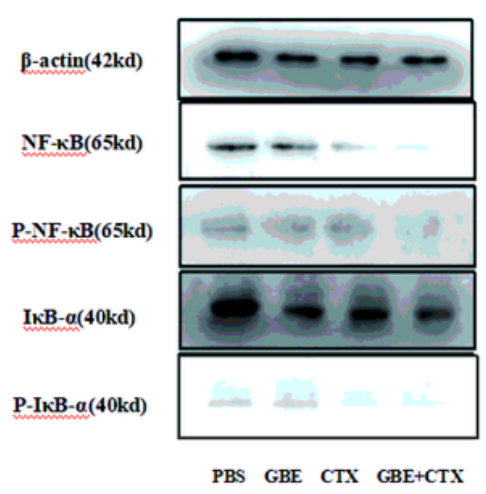

$\mathrm{C}$

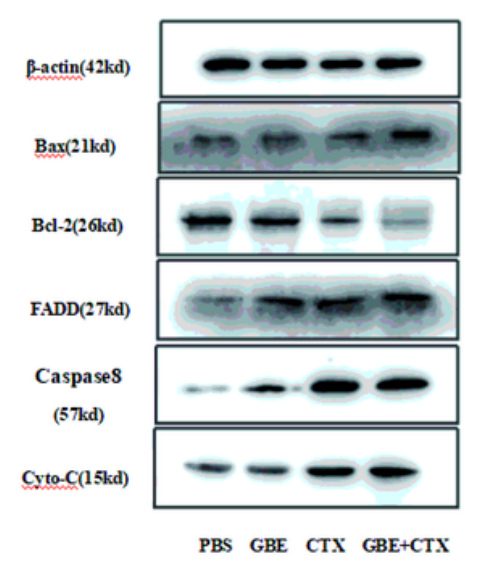

E

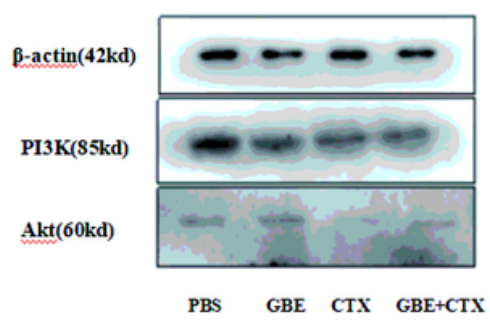

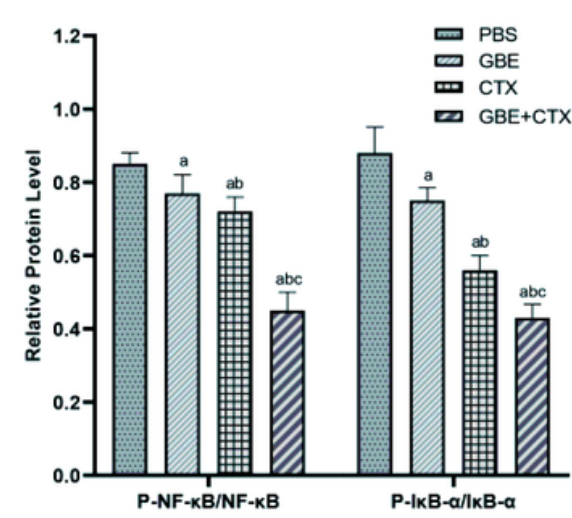

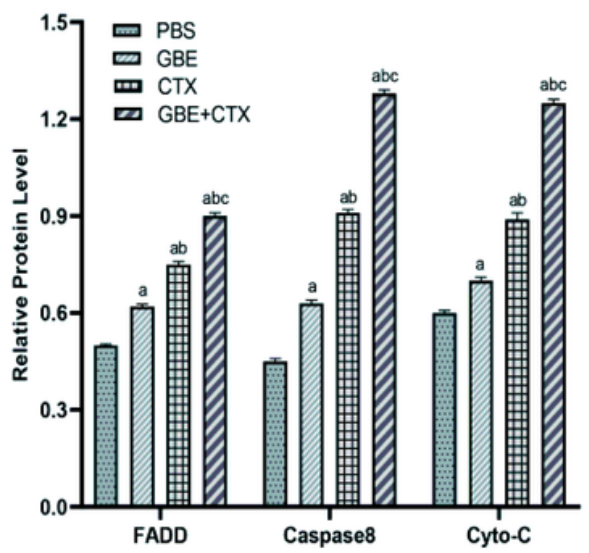

$\mathrm{F}$

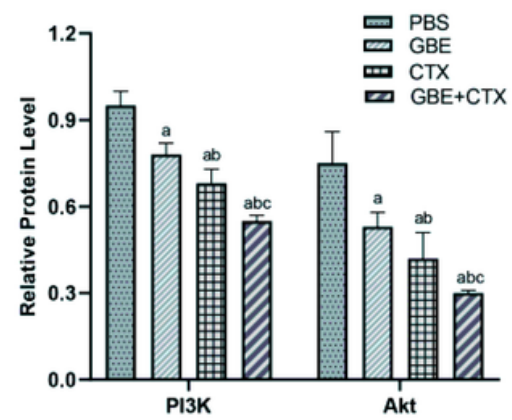

B

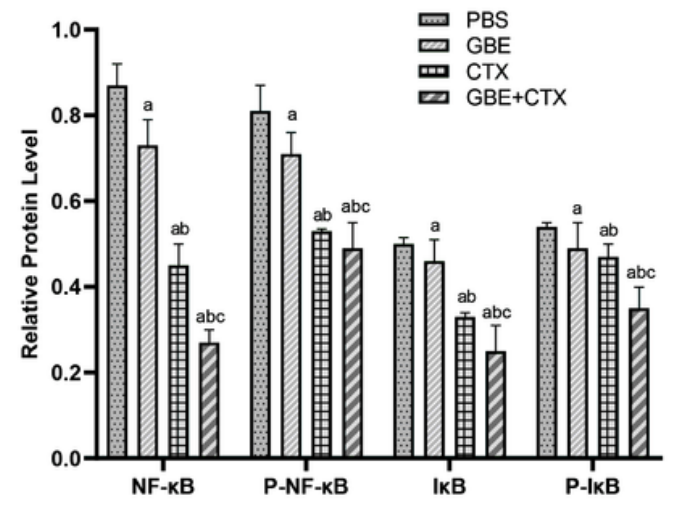

D

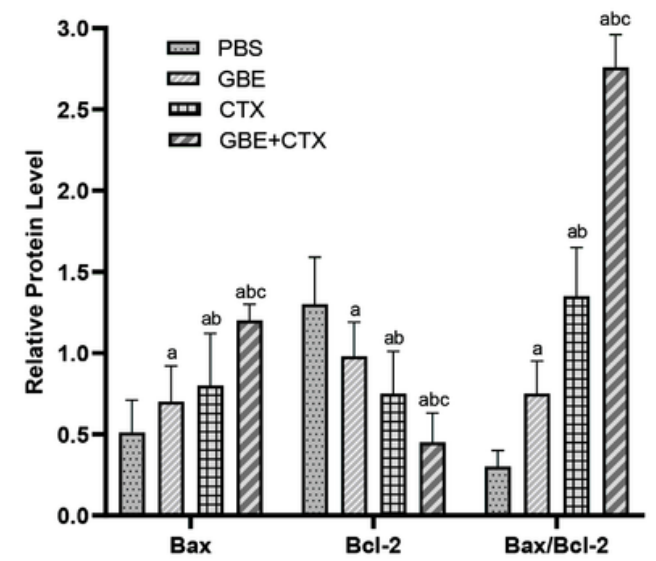

Figure 6

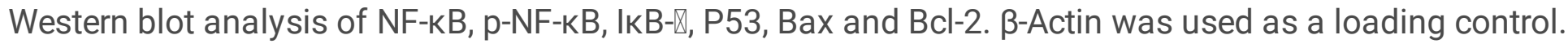
Representative images from three independent experiments and the results of densitometric analyses (means \pm SDs) are shown. Full-length blots/gels are presented in Supplementary Figure (Original data for western blot gel). The photographs generated were quantitatively analyzed with a Quantity One image

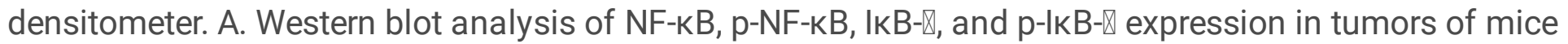
treated with PBS, GBE, CTX, or GBE+CTX. B. The comparison of NF-kB, p-NF-kB, and $p-I \kappa B-\otimes$ relative expression level in tumors of mice treated with PBS, GBE, CTX, or GBE+CTX. C. Western blot analysis and comparison of FADD, Caspase-8, and cyto-C expression in tumors of mice treated with PBS, GBE, CTX, or $\mathrm{GBE}+\mathrm{CTX}$. D. The comparison of $\mathrm{Bax}, \mathrm{Bcl}-2$ and ratio of $\mathrm{Bax}$ to $\mathrm{Bcl}-2$ relative expression level in tumors of mice treated with PBS, GBE, CTX, or GBE+CTX. E. Western blot analysis and comparison of PI3K and Akt 
expression in tumors of mice treated with PBS, GBE, CTX, or GBE+CTX. F. Western blot analysis and comparison of P53 expression in tumors of mice treated with PBS, GBE, CTX, or GBE+CTX. ap $<0.05$ vs PBS; $b p<0.05$ vs GBE; $c p<0.05$ vs CTX

\section{Supplementary Files}

This is a list of supplementary files associated with this preprint. Click to download.

- Originaldataforwesternblotgel.docx

- ARRIVEguidelines.docx 